\title{
Germanica
}

\section{Ungleichzeitigkeiten : Mentalitätswandel in der deutschen Literatur zur Einheit (1989-1999)}

Unification et évolution des mentalités dans la littérature allemande (1989-1999): des décalages dans le temps.

\section{Volker Wehdeking}

\section{(2) OpenEdition}

Journals

Édition électronique

URL : http://journals.openedition.org/germanica/2327

DOI : $10.4000 /$ germanica. 2327

ISSN : 2107-0784

Éditeur

Université de Lille

Édition imprimée

Date de publication : 30 décembre 1999

Pagination : $15-26$

ISBN : 9782913857018

ISSN : 0984-2632

\section{Référence électronique}

Volker Wehdeking, « Ungleichzeitigkeiten : Mentalitätswandel in der deutschen Literatur zur Einheit (1989-1999) », Germanica [Online], 25 | 1999, Online erschienen am: 28 Januar 2014, abgerufen am 06 Oktober 2020. URL : http://journals.openedition.org/germanica/2327 ; DOI : https://doi.org/10.4000/ germanica. 2327

Ce document a été généré automatiquement le 6 octobre 2020.

(ㄷ) Tous droits réservés 


\title{
Ungleichzeitigkeiten :
} Mentalitätswandel in der deutschen Literatur zur Einheit (1989-1999)

\author{
Unification et évolution des mentalités dans la littérature allemande \\ (1989-1999): des décalages dans le temps.
}

\section{Volker Wehdeking}

1 Die Nachwendeliteratur seit 1989 setzt sich auf dem Wege zur Berliner Republik mit dem Stadtroman, mit der Nacht des Falls der Mauer und mit dem Weg zu den Montagsmärschen auseinander. Es gab schon vor der Wiedervereinigung, seit Mitte der 60er Jahre (bei Christa Wolf und Günter Kunert etwa) und zunehmend seit 1971, Kritik an den eigenen Zuständen in der DDR, die Erich Honecker scheinbar zu dulden schien, bis mit der Ausbürgerung Wolf Biermanns Ende 1976 der Exodus der meisten wichtigen Autoren in den Westen einsetzte. Nach 1980 näherten sich die Themen und Schreibweisen in West und Ost einander allmählich an, auch wenn sie noch kein gemeinsames kulturelles Feld bildeten: Nachrüstung, Frauenemanzipation und neues Umweltbewußtsein (etwa in Monika Marons Flugasche, 1981) wurden in beiden Deutschland thematisiert, und in der DDR zeigten sich zunehmend auch ästhetisch Ansätze zu westlicher Spätmoderne.

Die kurze Euphorie nach dem Fall der Mauer hat auch in der deutschen Literatur einem breiten Mißvergnügen der Ungleichzeitigkeiten Platz gemacht. Diese zehn Jahre deutscher Romane seit 1989 stehen im Zeichen des Umbruchs auf dem mühsamen Weg zur kulturellen Reintegration beider Deutschland. Die Auffächerung der Traditionsstränge in Mitteldeutschland seit etwa 1985 läßt sich den Aporien der frühen Nachkriegsliteratur (1945-1952) in einer Kontra-faktur vergleichen: was damals getrennt wurde, wächst nun, ungeachtet aller Rückschritte, wieder zusammen. Der moderne Berlinroman west- und ostdeutscher Autor(inn)en, frauenspezifische und ökologiebewußte Texte herrschen neben dem System- und Generationenwandel ebenso vor wie das neue Unbehagen an der Ellbogengesellschaft. 
Das läßt sich an den aus der ehemaligen DDR kommenden Lyrikern Volker Braun, Günter Kunert und Durs Grünbein als Mentalitätswandel ebenso ablesen wie die neueste Skepsis gegenüber einer zunächst herbeigesehnten Integration. Einen ähnlichen Wandel zeigen Romane und Dramen junger Autoren wie Kurt Drawert, Thomas Brussig, Ingo Schulze und im Westen neue Berlinromane von Peter Schneider und Jakob Arjouni. Generationenromane von Monika Maron, Brigitte Burmeister, Wolfgang Hilbig und Reinhard Jirgl, aber auch Autobiographien von Günter de Bruyn, Christoph Hein, Günter Kunert und Martin Walser ließen in den späten 90er Jahren die Nachkriegsentwicklung wiederaufleben. Kurz vor der Epochenschwelle des Jahres 2000 liest sich dies auffallend parallel zu den Familien- und Generationenromanen in den USA (Tyler, Updike, De Lillo und Roth), Italien (Tamaro) und Frankreich (Rouaud).

Die Rolle gerade des West-Fernsehens für die Stimmungslage der DDR-Bevölkerung in den Jahren vor der Wende ist sicherlich ein mitentscheidender Faktor für die Entwicklung von 1989 gewesen. Das betont der renommierte Historiker Eberhard Jäckel in seiner neuen Studie Das deutsche Jahrhundert (1996) zum Dilemma einer DDR zwischen 1961 und 1989, nur durch eine Mauer ihre Bevölkerung an der Flucht hindern zu können, aber die Medien nicht mitauszugrenzen :

Die Absperrung, die allein sie sichern konnte, hatte jedoch den fast ebenso existentiellen Nachteil, vor aller Welt offenkundig zu machen, daß dieser Staat vom Volk nicht legitimiert war. Insofern die DDR nur ihren eigenen Bürgern, von streng geprüften Ausnahmen abgesehen, die Ausreise verwehrte, fremden Personen aber weiterhin grundsätzlich die Einreise gestattete, rückte sie sich selbst in den Rang eines Gefängnisses, dessen Insassen es nicht verlassen, wohl aber Besuch empfangen dürfen. [...] Die DDR verbot zwar die Einfuhr von Zeitungen und Zeitschriften. Den Versuch aber, den Bürgern auch den Empfang von westdeutschem Rundfunk und Fernsehen zu verbieten, mußte sie nach einiger Zeit aufgeben. Die Folge war, daß die Bevölkerung oder doch der größte Teil von ihr ständig gleichsam aus ihrem Gefängnis hinausblicken konnte. Kein anderes Regime in der Welt mußte unter so schwierigen Bedingungen regieren ${ }^{1}$.

5 So kann Eberhard Jäckel zu dem Fazit kommen, daß die DDR, in ihrem Bemühen seit 1949, sich von dem alten Staat, also dem besetzten Deutschland, zu distanzieren, nie über die Rolle eines «Gegenstaats im Osten», und damit ohne «wirkliche Eigenständigkeit» hinausgelangt war².

Nicht nur die Bewohner blickten ständig nach Westen. Die ganze Geschichte der DDR stand unter dem Gegensatz zur Bundesrepublik. Bereits ihre Gründung am 7. Oktober 1949 war eine Entgegnung auf die deutsche Staatsgründung [...] am 23. Mai 1949. [...] Wirkliche Eigenständigkeit war ihr niemals vergönnt ${ }^{3}$.

$\mathrm{Da}$ es noch im selben Monat November 1989 jedoch $\mathrm{zu}$ jenem entscheidenden Parolenwandel kam, von «Wir sind das Volk» zu jenem Wunsch nach deutscher Einheit in der Parole "Wir sind ein Volk», und in weiteren Wahlen zum Beitritt zur Bundesrepublik, zur Übernahme ihres Namens und Grundgesetzes, mit der allerdings gewichtigen einzigen Neuerung des Wechsels von Bonn nach Berlin, hat gewiß auch kulturelle Traditionsgründe, eine weitgehende Kontrafaktur mit vergleichbaren kulturellen Komponenten solcher Gegen-Spiegelung. Die Nachwendeliteratur reicht von 1990 bis heute, mit Vorläufersymptomen seit etwa 1980, als ein allmähliches Zusammenwachsen im «literarischen Feld» anhand der Formen und Themen. Die beiden Währungsreformen, der 20. Juni 1948, gefolgt von der Berliner Luftbrücke 1948/49, und der 1. Juli 1990 mit der neuen Wirtschafts- und Währungsunion sind ebenso Scharniere der Darstellung einer solchen Spiegelung, wie die staatliche 
Trennung und Wiedervereinigung: die Gründung der beiden Deutschland 1949 (Mai und Oktober) und - nach der Maueröffnung am 9. Nov. 1989 - der 3. Oktober 1990 als Beitritt der DDR zur Bundesrepublik und deren Grundgesetz nach Artikel 23.

Das Jahr 1990 wurde das erstaunlichste des Jahrhunderts. Nie zuvor hatte sich in Deutschland so vieles so grundlegend, so friedlich, so freundlich und so einvernehmlich geändert [...] auf zwei Ebenen, einer deutschen und einer internationalen, die wechselseitig aufeinander einwirkten ${ }^{4}$.

7 Die gelenkte, zensierte und geheimdienstlich überwachte Verlags- und Medienszene machte buchstäblich über Nacht der freien Marktwirtschaft Platz. Was aus DDR-Sicht in der ersten Nachwendezeit wie die «Furie des Verschwindens $»^{5}$ gewirkt haben mochte, so daß Volker Braun klagte, «die eine Struktur schiebt sich über die andere wie Lava ${ }^{6}$, sollte bald einer neuen, auch zahlenmäßig eindrucksvollen Blüte weichen. Heute sind aus der Subkulturszene Dresdens, Leipzigs und Ostberlins erwachsene Verlagsneugründungen, zusammen mit dem Erhalt umgestalteter alter Verlage und neuer Foren der Bürgerbewegungen zu einer Zahl von 250 Verlagen in den neuen Bundesländern im Jahre 1995 - gegenüber 78 Verlagen vor 1989 - angewachsen. Auch den alten Kulturhäusern und Jugendclubs, die in ländlichen Gebieten zu DDR-Zeiten zu einer beispielhaften Kulturdichte geführt hatten, stehen trotz des marktbedingten Kahlschlags besonders in Ostberlin allein bis 1993 «an die 500 neu gegründete Kulturvereine und -initiativen $»^{7}$ darunter viele Galerien, gegenüber. Freilich müssen die neuen Bundesländer mit ihrer immer noch höchsten Theaterdichte im weltweiten Vergleich ihre vor 1989 etwa 6500 neuen Buchtitel im Jahr nun in die Konkurrenz mit bundesdeutschen 60.000 Neuerscheinungen einbringen. Renommierte Zeitschriften wie «Sinn und Form» konnten gerettet werden (heute im konsolidierten Aufbau-Verlag erscheinend), andere wie «Neue deutsche Literatur» oder «Theater der Zeit» erlebten drastische Abonnenten-Einbußen, von 14.000 auf 2.500 bzw. 12.000 auf 3.500 im Jahre 1992. Das deutliche Wiedererstarken der Leipziger Buchmesse ist ein weiteres, innovatives Hoffnungszeichen, wie Medienresonanz und Feuilleton-Sonderbeilagen seit dem Frühjahr 1997, etwa in der «Zeit» und der FAZ, eindrucksvoll, auch im Bereich der Belletristik, belegen.

8 Nach 1989 und besonders Mitte der 90er Jahre häufen sich DDR-Aufarbeitungstexte in spätmoderner Ästhetik und neuer Satire mit Leserwirkung in den «alten» und «neuen» Ländern. Daß nun, seit 1994 zunehmend, Satireformen und Humor in die deutschdeutsche Thematik oder die DDR-Generationen- und Aufarbeitungsthematik Einzug halten, ist ein Zeichen für die allmähliche Normalisierung im Kulturbereich ungeachtet weiterbestehender Ungleichzeitigkeiten. Rita Kuczynskis neuer Roman Staccato (1997) etwa spiegelt die derzeit abwartende Stimmung zwischen West und Ost in der Skepsis einer jungen Frau angesichts der Erfahrungen mit dem raschen Anschluß an den Westen; sie resigniert ungeachtet eines nach der Wende ihr zugefallenen Ost-Berliner Hauses und verliert ihr Erbe wieder; die vermeintliche Chance der Wende erweist sich einmal mehr als Identitätsverlust. Thomas Brussig findet mit seinem schwarzem Humor im Roman und neuerlich der Bühnenversion von Helden wie wir (1995) ein breites Publikum in West und Ost. Der Selbstbefreiungstext Abgehauen von Manfred Krug ebenso wie Margarethe von Trottas Mauer-Film «Das Versprechen» (1995) oder Erich Loests Fernsehverfilmung «Nikolaikirche» durch Frank Beyer zeigen Stationen auf dem Weg zur Wende und Jurek Beckers Fersehserie «Wir sind auch nur ein Volk» (1994/95) konfrontiert die ungleichen Mentalitäten mit trockenem Understatement. Die ästhetischen Mittel von Brussig, Drawert und Kuczynski verweisen auf die 
Wiederaufnahme westlicher Spätmoderne. Einer Erzählprosa voll innovativer Kontraste wie jene des 1962 in Dresden geborenen Ingo Schulze über die Menschen in St. Petersburg, 33 Augenblicke des Glücks (1995), kann man, in den Worten des Bremer DDR-Literaturhistorikers Wolfgang Emmerich, kaum mehr « das Etikett DDR-Literatur » anheften : «Hier hat ein Autor einen Text jenseits aller DDR-typischen Lebens- und Schreibmuster vorgelegt (und seien es die des Prenzlauer Berges) - und damit signalisiert, daß DDR-Literatur natürlich nach und nach aufhört» ${ }^{8}$.

Einen etwas anderen Ansatz als Emmerich vertritt Iris Radisch in der Zeit ${ }^{9}$, wenn sie bereits im Untertitel ihres Beitrags zu beiden deutschen Literaturen für 1997 und vor allem im Blick auf Reinhard Jirgls Roman Hundsnächte (1997) mit dessen Arno Schmidt nahen experimentellen Zahlenzeichen und Expressionismen in der Landschaftsgestaltung oder der gedrängten Syntax die zugespitzte These vertritt: « immer noch, jetzt erst recht, gibt es zwei deutsche Literaturen: selbstverliebter Realismus im Westen, tragischer Expressionismus im Osten. «Da aber war der von einem breiten Spektrum der deutschen Feuilletonkritik als bedeutend eingestufte, neue Roman von Ingo Schulze, Simple Stories (1998), noch nicht erschienen, und der inzwischen ebenfalls vielbeachtete «Wenderoman» (Martin Walser) ${ }^{10}$ von Gert Neumann, Anschlag (1999) belegt ebenfalls, daß kein «tragischer» Expressionismus dominiert, wohl aber gelegentlich, wie auch bei Angela Krauß (Die Überfliegerin, 1995), eine Wiederaufnahme von Kafka. Wenn man überhaupt von einer ostdeutschen Dominante im Erzählen am Jahrhundertende sprechen darf, wo soviele Stile und Sichtweisen auftreten, dann ist sie eher in der Zuwendung $\mathrm{zu}$ neueren angloamerikanischen ästhetischen Strukturen $\mathrm{zu}$ finden, vom Minimalismus der Alltagsschilderung in den szenischen Fragmenten bei Sparschuh, Schulze, und LangeMüller bis zur Philip Roth verpflichteten Satire Thomas Brussigs und zum psychologischen Realismus der Burmeister und späten Christa Wolf (Hierzulande Andernorts. Erzählungen, 1999).

10 Für den neuen Roman von Ingo Schulze, Simple Storys, muß man diese Einsicht noch einmal differenzieren : die Lebensgeschichten der Altenburger sind sehr wohl typisch für die "neuen Bundesländer" neun Jahre "danach" : sie zeigen die Schwierigkeiten der neuen Identitätsfindung im kulturellen Vorgang einer zunehmenden «Verwestlichung des Ostens». Schon der Titel Simple Storys. Ein Roman aus der ostdeutschen Provinz (Berlin : Berlin Verlag, 1998) verrät einiges über die ungewöhnliche Machart des Romans : es sind 29 Kurzgeschichten, erzählt als Alltagsausschnitte und locker verbunden durch rund zwei Dutzend wiederkehrender Romanfiguren, die sich um die Familien Meurer und Schubert kristallisieren.

11 Wir erleben Durchschnittsbegebenheiten aus Altenburg bei Leipzig, wo Schulze (geb. 1962) in den frühen 90er Jahren Dramaturg am Landestheater und Redakteur war. Die "simple" Oberfläche täuscht : der "Untertext" zeigt, daß nach der Wende manchen im täglichen Überlebens- und Anpassungskampf die Vergangenheit nicht schlafen läßt und soziale Positionen sich verschieben wie auf dem Glücksrad. Einzig die Gemeinschaft bleibt, wie ehedem gefestigt, auch wenn es zu Totschlag, Scheidung und Vergewaltigung kommt. Die Szene bleibt, wie im falschen Plural «Storys», provinziell, Nebenschauplätze sind auch einmal Berlin, Assisi und New York. Die Vielschichtigkeit der Handlungen des großes Personenensembles ist so angelegt, daß es keine eigentlichen Hauptfiguren gibt, nur ein Kaleidoskop verfehlter Glücks- und Identitätssuche. 

während der DDR-Jahre als Schuldirektor Übermacht verlieh, und seines einstigen Opfers, dem Lehrer Dieter Schubert, den er zu Berufsverlust und drei Jahren Zwangsarbeit wegen des Duldens «antibolschewistischer» Sprüche brachte, umfaßt neben der Pädagogik Journalist(inn)en, Kunsthistoriker, Kellner, Hausfrauen, Schriftsteller, Politiker und - natürlich - den westlichen Immobilienspekulanten und Manager. Das Ganze wird geboten in Perspektivenwechseln und harten Schnitten wie im Film, ungeachtet knapper Inhaltsvorblenden. «Der rote Meurer» wird nun, nach seiner Bloßstellung durch Dieter Schubert - in einer grotesken Ansprache von der Kirchenmauer in Assisi herab -, von allen in die Isolation und zunehmende Paranoia getrieben. Schuberts Familienleben gerät nach der Wende ebenso auf Abwege: die Tochter Conny wird von einem West-Spekulanten vergewaltigt, er selbst läßt die Ehefrau zugunsten einer quasi Prostituierten im Stich und erleidet einen Herzinfarkt beim Karpfenangeln. Alle Personen werden nach der Wende in ihrer bürgerlichen Existenz beschädigt: so arbeitet der Kunsthistoriker nun als Froschmann in der Fußgängerzone, seine Frau stirbt, weil man sich kein Auto mehr leisten kann, auf dem Fahrrad, angefahren vom Wagen einer Politikergattin, die meint, es sei nur ein Dachs gewesen und keine Hilfe leistet. Die Direktorin des Naturkundemuseums verliert die Stellung, vielleicht wegen Stasi-Mitarbeit, und probiert voller Lebensgier die Männer durch, gerät an den Alkohol und heiratet schließlich fast wahllos. Die Warenwelt bleibt greifbarer als die Partnerbeziehungen, der einzige Schriftsteller im Roman begeht Selbstmord, aber kunstvoll enthält sich der Autor jeden psycholo-gischen Kommentars. Die Kritik hat gerade an diesem Verfahren die Qualität eines seismographischen Nachwenderomans gefeiert und Altenburg als Paradigma einer Welt bedrohter Lebenssicherheit im Umbruch erkannt.

Thomas Brussigs Roman Helden wie wir (Berlin: Vlg. Volk und Welt, 1995) holt die internationale Spätmoderne durch Anlehnungen an Philip Roth, John Irving und Günter Grass' Blechtrommel (1959) nach. Sein noch im selben Jahr in zweiter Auflage erschienener, inzwischen auch als Theaterstück aufgeführter, Schelmenroman zeigt einen Antihelden Klaus Uhltzscht, der seine Kindheit in einem alle Sextriebe unterdrückenden Elternhaus - die Mutter ist Hygieneinspektorin, der Vater bei der Stasi - als Außenseiter erlebt.

Zum Ende der Mauer kommt es, als die abwartenden Mitbürger an einem offenen Grenzübergang noch zögern, wer vorangehen soll; da stürmt Uhltzscht in neuer IchStärke zum Tor, öffnet demonstrativ den Mantel, und die vom Anblick des gigantischen Glieds schockierten Grenzpolizisten lassen ihn wie hypnotisiert hindurch. Hinter ihm stürmt das Volk in die Freiheit. Gestützt auf die psychologische DDR-Studie von H.J. Maaz, Der Gefühlsstau, und Philip Roth' Portnoy's Complaint (1969) zeichnet der 1965 geborene Brussig seinen Schelm als "missing link" der DDR-Geschichte auf dem Weg zur Wende in einem satirischen Verfahren auf den Spuren von Rabelais. Über den auf die frühere Lustfeindlichkeit seiner Landsleute gemünzten Pikaro-Roman mit FreudAnspielungen und dessen pubertäre Komik konnten auch sie lachen, wie der Verlagserfolg dort zeigte. 


\section{Zwei Ansichten nach dem Fall der Mauer}

15 An der Christa-Wolf-Rezeption läßt sich - nach dem Literaturstreit 1990 - bis zur Aufnahme des jüngsten Medea-Romans (1996) die immer noch in Ost und West geteilte Bewertung für ein grundsätzliches Nachdenken und für eine behutsamere Verständigung auf beiden Seiten über die Rolle des nicht systemkonformen Autors in der DDR während der Diktatur fruchtbar machen. Wie Therese Hörnigk (Humboldt Universität) jüngst auf einem Bonner Symposion zur «Literatur in der Diktatur» ${ }^{11}$ ausführte, war Christa Wolfs Rolle als «Moralische Instanz» vor allem in der späteren DDR seit 1968 durchaus unbequem für den Staat :

In einer Wirklichkeit, in der Literatur in Ermangelung demokratischer
Öffentlichkeit als Transportmittel individueller und kollektiver
Selbstverständigung fungierte, ließ sich gerade der moralische Gestus der Texte
lesen als Medium gegen Pragmatismus und autoritäre Machtanmaßung, der Werte
einklagte, die auf dem langen Wege einer fehllaufenden Geschichte
verlorengegangen oder nie in Angriff genommen waren. In dem Maße, wie die
"offizielle Wahrheit" alleinige Geltung beanspruchte und gleichzeitig mit der
Erfahrung der Individuen in Widerspruch geriet, begannen auch in der DDR
kritische Intellektuelle, die Wirklichkeit an der Idee zu messen ${ }^{12}$.

Der öffentliche Protestbrief gegen die Biermann-Ausbürgerung, von Hermlin und Stefan Heym initiiert und auch vom Ehepaar Wolf unterschrieben, markierte 1976 diesen Umschlag, der im Rückblick ein kulturelles Signal für den Anfang vom Ende der DDR bedeutete. Ein anderes Thema ist es jedoch, ob noch 1996, mit Christa Wolfs MedeaRoman, ein Beharren auf der eigenen früheren Position, wie sie in den Reformvorschlägen im Aufruf «Für unser Land» am 4. November 1989 laut wurde, in allegorischer Verbrämung überzeugen kann. Eine aufrichtigere Aufarbeitung der DDRJahre gelingt dagegen Günter de Bruyn in seinem neuen «Lebensbericht» Vierzig Jahre (1996).

In diesem, passagenweise doch sehr dicht und intensiv geschriebenen Lebensbericht, ist Günter de Bruyns Ehrlichkeit in der Entscheidung für die Innere Emigration in der DDR auffallend. Seine Anhänglichkeit an die Ostberliner Bibliotheksjahre, den Wohnsitz der Mutter und ein billig erworbenes Bauernhaus als Schreib- und Fluchtort an einem Nebenflüsschen der Spree, zwei Bahnstunden von Berlin entfernt, geben ihm «Bodenhaftung $»^{13}$, er hält aber unbeirrt am Fortbestand einer deutschen Kulturnation während all der DDR-Jahre fest und empfindet schließlich den Fall der Mauer als ein Stück glücklich verlaufener deutscher Geschichte, hierin am ehesten Martin Walser vergleichbar. In der frühen Nachkriegszeit noch leidenschaftlicher Thomas-MannLeser und die Gruppe 47, darunter lebenslang Heinrich Böll, mit Interesse verfolgend, kann sich de Bruyn der Kulturbürokratie lange entziehen, da sein Interesse an Jean Paul, dessen Biographie er unter anderem schreibt, und seine Erzählungen der leisen Töne mit Wanderungen in der Mark auf den Spuren Fontanes nicht auffallen. Bis in die siebziger Jahre hinein stellt er gerade am Beispiel des Vorbilds Böll an sich selbst diesen Mangel deutlicherer Zeitkritik fest :

Bedeutet nicht Böll-Nachfolge in diesem Teil Deutschlands, so hätte ich fragen können, den hiesigen Herren, statt sie zu preisen, die Wahrheit zu sagen? Aber was ich dann sagte, fiel harmloser, schwerer verständlich und vor allem viel länger aus $^{14}$.

[...] Waldeinsamkeit ist mir nie unheimlich gewesen. Immer waren es Menschen, die mich erschreckten. Denen war ich nun ausgewichen. Es war das Glück, den 
erträumten Rückzug geschafft zu haben, der mich nicht schlafen ließ. Ich war, dachte ich, in die Emigration gegangen, ohne das Land, das mich hielt, verlassen zu haben. Dem Staat war ich auf seinem eigenen Territorium entflohen. Hier würde es mir besser gelingen, die Zensur beim Schreiben aus meinem Bewußtsein zu tilgen. «De Bruyn beschreibt aber auch mit bitterer Ironie, wie noch in der selben ersten Nacht im neuen Refugium "Panzerspähwagen" zu einem dreitägigen Manöver anrollen,» als wollten sie mich Staatsräson lehren. Die Russen bringen Granatwerfer in Stellung und «Tag und Nacht lärmten Panzer und Tiefflieger. Infanteristen stürmten brüllend durch meinen Garten, ohne mir auch nur einen Blick zuzuwerfen ${ }^{15}$.

18 Als de Bruyn 1976 das Biermann-Protestschreiben mitunterzeichnet, wird die Waldidylle öfters von der Stasi heimgesucht, die mit bösen Tricks wie der Verleumdung eines mit fingierten Fotos bewiesenen Ehebruchs mit einer Pfarrersfrau aus der katholischen Bewegung dem standhaften Autor zusetzen, vergebens. Am Ende der DDR, ein halbes Jahr vor der Maueröffnung, soll er den Nationalpreis erster Klasse erhalten, dessen Erhalt bisher Kurt Hager verhindert hatte, und nun hat de Bruyn den Mut, den Preis abzulehnen. Lapidar notiert er: «Die Souveränität, die ich Spätentwickler gewonnen hatte, war dem Staat inzwischen abhanden gekommen. Vierzig Jahre lang war er mit Hilfe von Angst regiert worden ; als die sich abgebaut hatte, war es mit dem Regieren vorbei» ${ }^{16}$.

Die Begriffsklammer DDR-Literatur sollte daher «zwischen den beiden Polen der Affirmation und der radikalen Dissidenz angesiedelt» werden ${ }^{17}$. Hier, wo die großen literarischen Debatten in der DDR weiterreichende Wirkung zeigten und aus dem «Spannungsverhältnis zwischen Erziehungsdiktatur und künstlerischem Wollen» der einzelnen Autoren produktive Kraft jenseits von Affirmation herauskam, die im Kern «kritisch und subversiv» war, ergibt sich «ein weiterwirkender Kanon mit den Namen Christa Wolf und Heiner Müller an erster Stelle, danach Jurek Becker, Günter de Bruyn, Fritz Rudolf Fries, Christoph Hein, Stephan Hermlin, Stefan Heym und Volker Braun, sicher auch Peter Hacks; darüber hinaus alle ausgewanderten oder ausgewiesenen Autoren mit DDR-Themen, wie Sarah Kirsch, Günter Kunert, Monika Maron und Erich Loest ${ }^{18}$. Die Jungen der 80er Jahre, auch die Stasi-Verstrickten unter ihnen, tragen mit der Aufarbeitung ihres Mißvergnügens mit dem Staat oder in der Prenzlauer Berg Postmoderne zu diesem lebendigen, auch mentali-tätsgeschichtlich faszinierenden und oft provozierenden Spannungsverhältnis der DDR-Literatur zur Staatssicherheit bei, für mich am eindrucksvollsten im Roman Wolfgang Hilbig, im Drama Christoph Hein, in der Lyrik Durs Grünbein, im Essay Kurt Drawert.

Der Mentalitätswandel von der Befreiungseuphorie zum Alltag skeptischen Abwartens zeigt sich deutlich im Vergleich zwischen dem pathetisch expressiven Gedicht von Durs Grünbein von 1989, noch vor der Maueröffnung entstanden, mit dem sprechenden Titel : «O Heimat, zynischer Euphon» (20.3.1989) und dem leisen, melancholischen Drama von Kurt Drawert von 1996 mit dem Titel Alles ist einfach (im Juli 1996 in Darmstadt uraufgeführt). Der Büchner-Preisträger Grünbein (geb. 1961) betonte im März 1989, als niemand den baldigen Fall der Mauer ahnen konnte, noch das Trennende beider Kulturen, aber auch die spiegelbildliche Abhängigkeit im Bild des «Doppel-Klons», das E. Jäckels Einsicht in den «Gegen-Staat» DDR bestätigt. Sein Gedicht ist zusätzlich angereichert mit nicht geheurer Tradition durch die BennParodie im widersprüchlichen Künstler-Selbstverständnis wie im Hinweis auf die gemeinsame Erinnerung an die «Bombensplitter» des Zweiten Weltkriegs : 
[...] «Noch Bombensplitter ? !» Gut für Stachelgaumen, in violetten Babyschädeln installiert

Sag, welche Schwester drückte ihren Daumen

Ins zarte Fontanell uns ungerührt?

Geröntgt, geimpft, dem deutschen Doppel-Klon,

Gebrochnen Auges, das nach Weitblick giert,

böse verfallen sind wir, pränatal dressiert.

«Deutschland?»... O Heimat zynischer Euphon.

21 Kurt Drawert, im zornigen Essay-Band «Spiegelland» deutlich Sartre verpflichtet, verbindet im neuen Drama einen Nachhall von Beckett mit der Fortschreibung von Christoph Heins Ritter der Tafelrunde (1989). In einem brüchigen Krankenzimmer liegen zwei über 50jährige Patienten, Harry und Pit, Harry noch mit einer Fensteraussicht, aber nur auf die Hausmauer gegenüber. Ihr Warten auf etwas wird am Ende durch Soldaten und Müllmänner auf der Bühne beantwortet, die zwischen den Slogans «Der Krieg ist überall. Es lebe die Partei» und «Jetzt wird alles neu» zur radikalen Entleerung der Bühne schreiten.

Unter den Gespenstern der frischen Vergangenheit fällt in dieser Eiszeitatmosphäre der abgehalfterte König auf, im Gitterwagen eingesperrt, wirr und mit goldener Pappkrone im roten Bademantel. Ans Ohr hält er sich ein Tonbandgerät mit den eigenen pathetischen Reden an das unterdrückte Volk. Dann singt er "Good Bye Johnny» zur Melodie der Becher-Hymne und verläßt den Krankenraum vor den Soldaten und Müllmännern. Diese an Hilbigs Kunde von den Bäumen (1994) erinnernden Entrümpler sagen die zynischsten Parolen auf: «Jetzt wird alles neu, blühende Wirtschaft, blühende Landschaft, glückliche Hühner. Zukunft», sagen sie, und dazwischen fallen sie zurück ins gewohnheitsmäßige «Es lebe die Partei». Da sehen wir also Leute, die so vieles in Windeseile über Bord geworfen haben, daß es sie fast selber von den Planken gefegt hätte, wären da nicht diese wenigen kleinen Rettungsanker des Vergangenen, die heimeligen Parolen, die sich gegen die Entwurzelung stemmen.

«Wer sind wir eigentlich? Gibt es uns noch?» ${ }^{19}$ Diese Fragen weisen auf die schwierige Identitätssuche in einem nicht vordergründig politischen, aber die «inneren Verwerfungen der Zeit» auf «beklemmende Weise» spiegelnden Stück. Eine Phase spürbaren Unbehagens im täglichen Miteinander spiegelt sich hier, seit man die lange Dauer dieses wirtschaftlichen und kulturellen Zusammenwachsens ahnt, zusammen mit einer Spirale langandauernder Ungleichzeitigkeit, «von vielen, gerade im Westen, mit deutlicher Distanz und Selbstgerechtigkeit begleitet» ${ }^{20}$. In Peter Schneiders BerlinRoman Eduards Heimkehr (1999) um die Erfahrungen eines aus Kalifornien nach Charlottenburg 1996 zurückgekehrten Genforschers mit der veränderten Stadt ist der Antiklimax vielfacher Mentalitätskontraste im Stadtalltag nach dem als Traum vergegenwärtigten Jubel um die Maueröffnung in eine griffige Allegorie der « vorzeitig aus dem Leben gerissenen » DDR gefaßt :

Was er hörte, klang wie ein Nachruf auf eine geliebte [...] Person, deren Name jedermann bekannt war. Es mußte sich um eine außergewöhnliche Persönlichkeit handeln, eine schwierige, hochbegabte Frau - [...]. Anscheinend hatte die Betrauerte einen schrecklichen Fehler: Ihre Rechthaberei, ihre Unbelehrbarkeit, ihre Arroganz gegenüber dem Leben waren kaum auszuhalten gewesen. [...] Offenbar hatte sie sich nicht vorstellen können, von Geburt an von Feinden umgeben zu sein. [...] Schließlich hatten die Feinde die Überhand gewonnen und rissen nun in einem Plünderungszug ohne Beispiel alles an sich, was sie hinterlassen hatte - Seen und Häuser, die geistigen Liegenschaften, die Seele jener wunderbaren, schwierigen und geheimnisvollen Frau namens DDR ${ }^{21}$. 
Zehn Jahre nach der Maueröffnung bleibt gerade auch die kulturelle Reintegration offenbar eine ebenso lohnende wie schwierige Herausforderung. Die spätestens Mitte der 90er Jahre als eigenständige ästhetische Tradition ins bundesdeutsche literarische Feld aufgegangene DDR- Literatur wird ihre weiterschreibenden Autor(inn)en als Hinterbliebene eines gescheiterten Systems samt seinem Nachhall an Spannungen und Ungleichzeitigkeiten noch lange beschäftigen.

\section{NOTES}

1. Eberhard Jäckel, Das deutsche Jahrhundert. Eine historische Bilanz, Stuttgart 1996, S. 316 f.

2. Ebd., S. 308.

3. Ebd., S. 308 u. 319.

4. Ebd., S. 322.

5. Wolfgang Emmerich, Kleine Literaturgeschichte der DDR. Erweiterte Neuausgabe, Leipzig 1996, S. 436.

6. Ebd.

7. Ebd., S. 438.

8. Emmerich, S. 506.

9. Iris Radisch, «Der Herbst des Quatschocento», in : Die Zeit, 17.10.1997. Nachgedruckt in : A. Köhler/R. Moritz (Hg.), Maulhelden und Königskinder. Zur Debatte über die deutschsprachige Gegenwartsliteratur. Leipzig : Reclam 1998, S. 180-195.

10. Martin Walser, «Geist und Sinnlichkeit. Gert Neumanns deutsch-deutsches Gespräch». - Dazu auch: «Endlich! Der Wenderoman» über Gert Neumann : Anschlag. Köln : Dumont Buchverlag 1999.

11. Therese Hörnigk, Typoskript "Christa Wolf. Die Schriftstellerin als moralische Instanz», Symposion «Literatur in der Diktatur», Bonn- Bad Godesberg, Wissenschaftszentrum, 3. /4. Mai 1996. - Der Sammelband «Literatur in der Diktatur. Schreiben im Nationalsozialismus und DDRSozialismus», Günther Rüther (Hg.), Paderborn 1997, enthält ihren Beitrag nicht mehr.

12. Hörnigk, Wolf, Ms. Typoskript, S. 2.

13. Günter de Bruyn, Vierzig Jahre. Ein Lebensbericht, Frankfurt a. M. 1996, S. 261.

14. Ebd., S. 137.

15. Ebd., S. 159.

16. Ebd., S. 253.

17. Klussmann, Ms. Literatur in der Diktatur, S. 7.

18. Ebd.

19. E. Franke, Wolken ziehen vorüber, in : Stuttgarter Zeitung, 5. 7. 1996.

20. Ebd.

21. Peter Schneider, Eduards Heimkehr. Roman. Berlin : Rowohlt, 1999, S. 219 f. - Zur literarischen Traditionsbildung 1971 bis 1989 und den Nachwendejahren vgl. : Volker Wehdeking : Die deutsche Einheit und die Schriftsteller. Literarische Verarbeitung der Wende seit 1989. Stuttgart: Kohlhammer 1995. 


\section{RÉSUMÉS}

Die kurze Euphorie nach dem Fall der Mauer hat auch in der deutschen Literatur einem breiten Mißvergnügen der Ungleichzeitigkeiten Platz gemacht. Diese zehn Jahre stehen, ob im Gedicht, den wenigen Dramen und Filmen, vor allem den Romanen, im Zeichen des Umbruchs auf dem Weg zur kulturellen Reintegration beider Deutschland. Der Essay versucht, anhand der Studie von E.Jäckel, Das deutsche Hahrhundert (1996) und den Thesen von Günter de Bruyn zur Kulturnation die Westbezogenheit bereits der DDR-Medien für dir unumkehrbare Richtung des Wandels fruchtbar zu machen. An den Romanen von Thomas Brussig, Helden wie wir (1995), Ingo Schulze, Simple Storys (1998), der Autobiographie von Günter de Bruyn, Vierzig Jahre.Ein Lebensbericht (1996), der Christa-Wolf-Rezeption, Lyrik von Durs Grünbein und einem Drama von Kurt Drawert, Alles ist einfach (1996), zeigt sich der Wandel in der Generationenabrechnung und thematik zu neuer Skepsis und Ablehnung sowohl der Ellbogengesellschaft wie eines früheren Sozialismus «von oben». Das literarische Feld (Bourdieu) wird anhand des veränderten Literaturbetriebs nach 1990 und knapper Vergleiche mit der Trennungssituation der beiden Deutschland 1949 in seiner Integration umrissen.Ebenfalls skizziert werden Proleme der Literaturgeschichtsschreibung zur DDR-Literatur.

Après la chute du mur et une brève période d'euphorie, les rythmes différents des anciens et des nouveaux Länder se sont imposés dans les domaines littéraire et culturel.Ces dix années se caractérisent néanmoins dans tous les genres par une tendance à l'harmonisation. En se fondant sur Das deutsche Jahrhundert d'Eberhard Jäckel (1996) et les thèses de Günter de Bruyn sur la nation culturelle, cet article essaie de montrer comment déjà en RDA les médias étaient réactifs par rapport à l'Ouest. L'exemple des romans Helden wie wir de Thomas Brussig (1995), Simple storys de Ingo Schulze (1998), l'autobiographie Vierzig Jahre. Ein Lebensbericht de Günter de Bruyn (1996), la réception de l'œuvre de Christa Wolf, la poésie de Durs Grünbein et le drame Alles ist einfach de Kurt Drawert (1996), témoignent de l'évolution de la problématique des générations vers un nouveau scepticisme qui s'oppose tout autant à l'égoïsme de la société qu'au socialisme imposé « d'en haut».Cette étude esquisse une redéfinition du champ littéraire (Bourdieu) en se penchant sur ses nouvelles conditions de production depuis 1990, en les comparant brièvement à la situation des deux Allemagnes en 1949 et en évoquant les problèmes de l'histoire de la littérature de la RDA.

\section{AUTEUR}

\section{VOLKER WEHDEKING}

Hochschule für Bibliothek und Information Stuttgart 